\title{
21-cm observations and warm dark matter models
}

\author{
A. Boyarsky, ${ }^{1}$ D. Iakubovskyi, ${ }^{2,3}$ O. Ruchayskiy $\odot,{ }^{2}$ A. Rudakovskyi, ${ }^{3}$ and W. Valkenburg ${ }^{4}$ \\ ${ }^{1}$ Lorentz Institute, Leiden University, Niels Bohrweg 2, Leiden, NL-2333 CA, The Netherlands \\ ${ }^{2}$ Discovery Center, Niels Bohr Institute, Copenhagen University, Blegdamsvej 17, DK 2100, Copenhagen, \\ Denmark \\ ${ }^{3}$ Bogolyubov Institute of Theoretical Physics, Metrologichna Street 14-b, 03143 Kyiv, Ukraine \\ ${ }^{4}$ Institute of Physics, Laboratory for Particle Physics and Cosmology (LPPC), École Polytechnique \\ Fédérale de Lausanne, CH-1015 Lausanne, Switzerland
}

(Received 26 April 2019; published 9 December 2019)

\begin{abstract}
Observations of the redshifted 21-cm signal (in absorption or emission) allow us to peek into the epoch of the "Dark Ages" and the onset of reionization. These data can provide a novel way to learn about the nature of dark matter, in particular, about the formation of small-size dark matter halos. However, the connection between the formation of structures and the $21-\mathrm{cm}$ signal requires knowledge of a stellar to total mass relation, an escape fraction of UV photons, and other parameters that describe star formation and radiation at early times. This baryonic physics depends on the properties of dark matter, and in particular, in warm-dark-matter (WDM) models, star formation may follow a completely different scenario, as compared to the cold-dark-matter case. We use the recent measurements by EDGES [J. D. Bowman, A. E. E. Rogers, R. A. Monsalve, T. J. Mozdzen, and N. Mahesh, An absorption profile centred at 78 megahertz in the sky-averaged spectrum, Nature (London) 555, 67 (2018).] to demonstrate that when taking the above considerations into account, the robust WDM bounds are in fact weaker than those given by the Lyman- $\alpha$ forest method and other structure formation bounds. In particular, we show that a resonantly produced 7-keV sterile neutrino dark matter model is consistent with these data. However, a holistic approach to modeling of the WDM universe holds great potential and may, in the future, make 21-cm data our main tool to learn about DM clustering properties.
\end{abstract}

DOI: 10.1103/PhysRevD.100.123005

The hyperfine splitting of the lowest energy level of the neutral hydrogen atom leads to a cosmic $21-\mathrm{cm}$ signal thanks to the abundance of primordial hydrogen. The $21-\mathrm{cm}$ signal from the post-reionization Universe has been studied by a number of experiments (e.g., LOFAR [1,2], GMRT [3], PAPER [4] (see however [5]), MWA [6]), but the only tentative detection of the $21-\mathrm{cm}$ signal in absorption against the CMB background at $z \sim 16-19$ has recently been claimed by the EDGES experiment [7]. ${ }^{1}$ It is clear that the forthcoming experiments, such as the staged HERA [10] or future SKA [11,12], will offer detailed information about the distribution of the $21-\mathrm{cm}$ signal, thus allowing for the full 3D tomography of the signal, offering an unprecedented reach into the early Universe. This makes the study of the $21-\mathrm{cm}$ signal a promising tool to learn not only about cosmological parameters (see, e.g., [13-15]) but also about different properties of dark matter, including its decays and annihilations [16-21], dark matter-baryon interactions [22-27], and the formation of gravitationally bound structures [28-33].

In this work we focus on the global (sky-averaged) $21-\mathrm{cm}$ absorption signal that appears when the spin

\footnotetext{
${ }^{1}$ Note however that the result is still uncertain, and there are alternative, noncosmological explanations $[8,9]$.
}

temperature (logarithm of the ratio of population of two levels of the hydrogen's $1 S$ state) becomes smaller than the CMB temperature (for a review see, e.g., [29,32,34]). The standard explanation for this difference of temperatures is the presence of a bath of Ly- $\alpha$ photons which induce transitions between $1 S_{1}$ and $1 S_{3}$ levels: Ly- $\alpha$ pumping. Therefore, a detection of the global $21-\mathrm{cm}$ absorption signal at some redshift $z_{0}$ implies that sources of radiation have already been active at that epoch. With our current knowledge of baryonic physics, we can robustly state that such radiation sources can only form inside dark matter overdensities. Hence, to predict the $21-\mathrm{cm}$ signal, one has to follow several steps:

(a) Start from the description of bound gravitational structures at a given redshift $z$.

(b) Continue with the description of how baryons collapse into these structures (which depends both on the size of the structures, on redshift and on cosmology).

(c) Assuming a particular type of radiation sources (as they cannot be modeled from first principles), estimate the number of produced photons and model (usually through a combination of semianalytical and numerical methods) how radiation escapes from the bound structures and heats the ambient medium. 

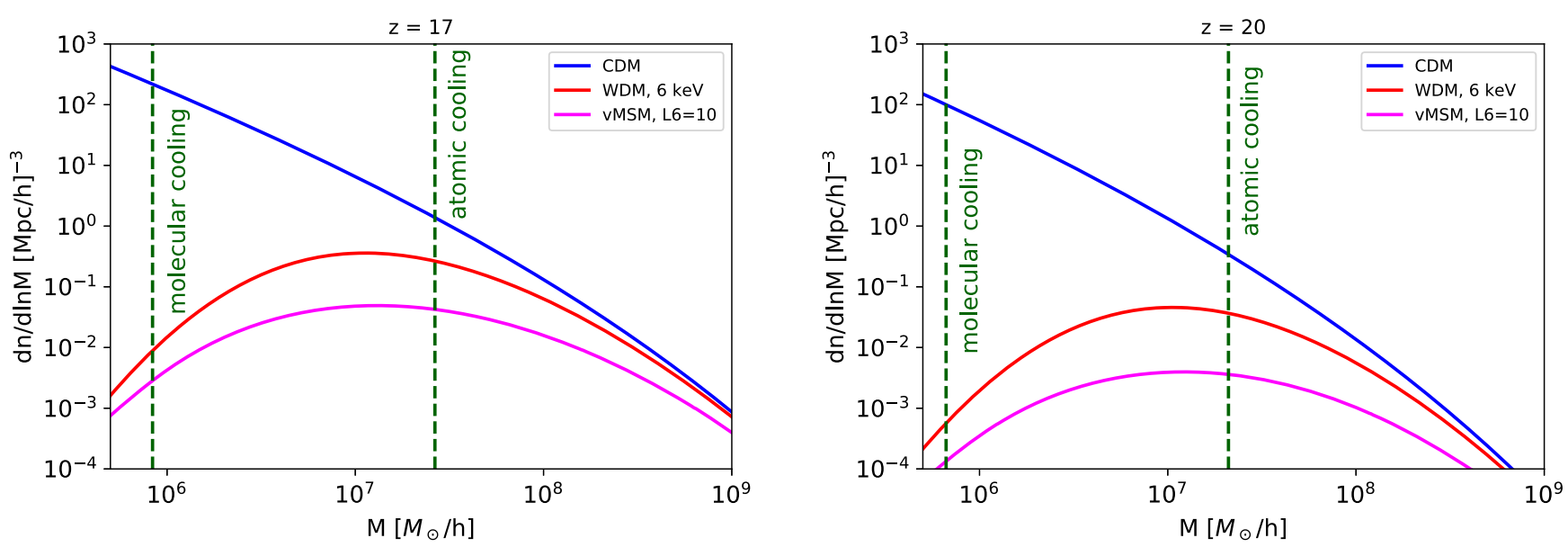

FIG. 1. Halo mass functions of models of our interest at redshifts 17 (left) and 20 (right). The masses that correspond to $T_{\text {vir }}=10^{3} \mathrm{~K}$ (molecular cooling) and $T_{\text {vir }}=10^{4} \mathrm{~K}$ (atomic cooling) are marked as green dashed vertical lines. At both redshifts the molecular cooling threshold has little effect on the collapsed fraction (1) in WDM and sterile neutrino models, while for CDM the impact of molecular cooling is substantial, as Fig. 5 illustrates.

(d) Given the resulting function of radiation density $d \rho_{\text {rad }} / d z$, one can then use available codes (such as ARES [35] or 21CMFAST [36]) to predict the 21-cm signal.

Uncertainties, as well as differences in predictions, between DM models are introduced at every step in this process.

(a) Bound DM structures.-Historically, the first warm dark matter models were those of sufficiently massive Standard Model neutrinos (see, e.g., [37]). Such particles were in thermal equilibrium in the early Universe and froze out while still being relativistic. They remained relativistic for some period in the radiation dominated epoch and homogenized primordial density perturbations on scales below the free-streaming horizon, $\lambda_{\mathrm{fs}}$ (for a proper definition see, e.g., $[38,39])$. The number density of such warm-dark-matter (WDM) thermal relics is uniquely determined by the temperature of freeze-out or, equivalently, by their mass, $m_{\mathrm{TH}}$. This mass of the thermal relic is the most typical parametrization of the WDM models. ${ }^{2}$ All WDM models have suppressed [as compared to cold dark matter $(\mathrm{CDM})]$ number of halos with masses below the freestreaming cutoff scale, $M_{\text {cut }}=\frac{\pi}{6} \lambda_{\mathrm{fs}}^{3}$, where $\lambda_{\mathrm{fs}}$ is the freestreaming horizon (see, e.g., [39]). This leads to a large difference between a number of collapsed halos, especially at high redshifts, between CDM and WDM models (see Fig. 1 for our halo mass functions calculated by using the standard prescription proposed in [42], also fully consistent with Fig. 1 of [43]). Naively, one could also expect a big

\footnotetext{
${ }^{2}$ An alternative parametrization is given by the mass of nonresonantly sterile neutrinos [40]. The two models lead to an almost identical shape of the matter power spectrum, and therefore their masses are related to one another in a nonlinear way; see [38,41] for details. In this work we always indicate what definition of mass we are using.
}

difference between two models in terms of produced starlight. However, only the halos with masses down to $10^{7}-10^{8} \mathrm{M}_{\odot} / h$ contribute to the formation of stars in CDM at redshifts of interest. Indeed, these masses correspond to virial halo temperatures $\sim 10^{3}-10^{4} \mathrm{~K}$-temperatures that are needed for the hydrogen to cool sufficiently fast, in order to collapse and form compact radiative sources [44,45]; see Eq. (2) below. In addition to halos, other bound DM structures-filaments-can exist in the early Universe. Near the cutoff mass formation of filaments and their subsequent fragmentation may be the dominant structure formation process in WDM [46,47], as opposed to the CDM model. The impact of filaments on the $21-\mathrm{cm}$ signal is studied by [48] (see also [49]), with the outcome that the lower bound on the WDM mass should be weakened compared with $\gtrsim 6 \mathrm{keV}$ in earlier works $[43,50]$ that did not take into account this effect. In addition to this difference, the presence of filaments also interferes with the structure formation processes, as discussed below.

(b) Baryonic collapse and star formation in different DM universes.-In general, the naive expectation that what is known from CDM simulations would also apply to WDM universes does not hold up. Let us point out two remarkable differences between star formation in CDM and WDM. First, in WDM universes star formation in filaments may dominate over star formation in halos at redshifts $z \gtrsim 6$ [46,51], producing different populations of stars and different amounts of Lyman- $\alpha$ photons. The star-formation efficiency of these processes is still highly uncertain, but it is clear that they can play a role. Such a mechanism is absent in CDM.

Second, both hydrodynamical simulations of galaxy formations (cf. [52-56]) and semianalytical models (cf. [57-62]) are tuned to reproduce galaxy observables (e.g., luminosity or stellar mass functions, etc.) at $z=0$. 
Not surprisingly, this leads to galaxy populations in CDM and WDM having similar properties in recent epochs [63]. However, in order to achieve this agreement, one has to choose quite different star-formation prescriptions in CDM and WDM at high redshifts [63], especially for halos close to $M_{\mathrm{fs}}$ [64]. As the halo formation in the WDM universe often starts later, one generically requires higher starformation efficiencies for WDM (consistent with what we infer in our work).

(c) Modeling radiation.-According to the well-developed theory of the $21-\mathrm{cm}$ signal in the early Universe (see, e.g., [32]), the key driver of the timing of 21-cm absorption is the emission rate of Ly- $\alpha$ photons that excite the electrons in hydrogen and result in a spin flip of such electrons after deexcitations (Ly- $\alpha$ pumping). The most common mechanism for emitting Ly- $\alpha$ photons at high redshifts is early star formation [32] (note however that the QSO contribution can also be significant; see, e.g., [65]). In a CDM universe, the bulk of stars is formed in halos. Therefore, the star-formation rate is usually parametrized by the ansatz (see, e.g., $[32,35,43,50,66]) \dot{\rho}_{*}(z)=f_{*} \bar{\rho}_{\mathrm{b}, 0} \dot{f}_{\text {coll }}(z)$, for redshift $z$, star density (calculated in comoving volume) $\rho_{*}, \cdot \equiv \frac{d}{d t}$ with time $t, \bar{\rho}_{\mathrm{b}, 0}$ the homogeneous baryon density today, $f_{\text {coll }}(z)$ the fraction of baryons in collapsed structures, and $f_{*}$ the fraction of collapsed baryons that form stars.

The fraction $f_{\text {coll }}(z)$ is derived from the halo mass function of a model as

$$
f_{\text {coll }}(z)=\frac{1}{\rho_{m}(z)} \int_{M_{\min }}^{\infty} d M \frac{d n}{d \ln M},
$$

with a cutoff for halos below mass $M_{\min }$ which are expected not to be able to form stars. This cutoff is set by the halo's virial temperature $T_{\text {vir }}$, the temperature which the gas reaches during the virialization of the halo [45]:

$$
\begin{aligned}
M_{\min }= & 1.0 \times 10^{8}\left(\frac{1+z}{10}\right)^{-3 / 2}\left(\frac{\mu}{0.6}\right)^{-3 / 2} \\
& \times\left(\frac{T_{\mathrm{vir}}}{1.98 \times 10^{4} \mathrm{~K}}\right)^{3 / 2}\left(\frac{\Omega_{m}}{\Omega_{m}^{z}} \frac{\Delta_{c}}{18 \pi^{2}}\right)^{-1 / 2} \mathrm{M}_{\odot} / \mathrm{h},
\end{aligned}
$$

where $z$ is the halo redshift, $\mu \simeq 0.60$ is the mean molecular weight, $\Omega_{m}^{z}=1-\Omega_{\Lambda} /\left[\Omega_{m}(1+z)^{3}+\Omega_{\Lambda}\right]$, and $\Delta_{c}=18 \pi^{2}+$ $82\left(\Omega_{m}^{z}-1\right)-39\left(\Omega_{m}^{z}-1\right)^{2}$ [67]. Depending on which mechanism is responsible for cooling, this cutoff may vary: atomic cooling is associated with a cutoff $T_{\text {vir }} \simeq 10^{4} \mathrm{~K}$, while molecular cooling leads to a cutoff $T_{\text {vir }} \simeq 10^{3} \mathrm{~K}$; see, e.g., Fig. 12 of [45]. The consequences of this parameter are discussed later and visualized in Fig. 1.

Galaxies or galaxy candidates have been observed for $z \lesssim 10$ [68], and we can only extrapolate the aforementioned ansatz for the redshifts of interest. The starformation efficiency in halos can be estimated from the observed ultraviolet luminosity function (UV LF) (see, e.g., [69-73]). The dependency $f_{*}(M, z)$ on halo mass and redshift relies on the model of star formation, and possible values of $f_{*}$ vary in a wide range. For example, in CDM halos $f_{*}$ may reach 0.3 at $z=5-8$ for $10^{11}-10^{12} \mathrm{M}_{\odot} / \mathrm{h}$ halos, increase with redshifts, and be close to unity during the Dark Ages [70]. In addition, the observational estimates of star-formation efficiency depend on assumed cosmology, and $f_{*}$ in low-mass galaxies may be higher in WDM compared to CDM (see, e.g., [74-76]). Apart from observations, $f_{*}$ can be predicted in CDM by use of detailed numerical simulations of the Universe during redshifts $z \sim 6-15$ [74,77-81]. However, there is a 3-orders-ofmagnitude scatter among the values of $f_{*}$ in individual simulated galaxies. As Figs. 15 and 16 of [78] demonstrate, a few galaxies with $f_{*} \simeq 0.3$ produce an amount of starlight which is several times larger than that of the bulk of galaxies with $f_{*} \simeq 0.01$. As a result, it is currently impossible to derive a robust constraint on $\dot{\rho}_{*}(z \sim 17)$.

An escape fraction of ionizing photons in galaxies during the reionization and the Dark Ages has not been determined directly and is still uncertain (see, e.g., Sec. 7.1 in [82]). However, varying the ionizing photon escape fraction in a wide range does not change the redshift of the $21-\mathrm{cm}$ absorption signal significantly. The escape fraction of photons in the band 10.2-13.6 eV is usually assumed to be close to unity (see Sec. 3.5 of [71] and references therein).

(d) Predicting the 21-cm signal.-The above-mentioned uncertainty on $f_{*}$ translates into a strong systematic uncertainty on WDM parameters that can be probed with a 21-cm absorption signal. In order to demonstrate this, we computed the $21-\mathrm{cm}$ absorption signal using the ARES code for three models: CDM, thermal relics with a mass $m_{\mathrm{TH}}=$ $6 \mathrm{keV}$ (claimed to be excluded in $[43,50]$ ), and the resonantly produced sterile neutrino, with particle mass of $7 \mathrm{keV}$ and lepton asymmetry $L_{6}=10 .^{3}$ This sterile neutrino model is consistent with all astrophysical and cosmological bounds: x-ray bounds on decaying DM [39,85-92], suppression of the power spectrum as inferred from the Lyman$\alpha$ forest [93-95], cosmic reionization [64,96,97], and Milky Way satellite and galaxy counts [56,61]. The results are shown in Fig. 2. The results strongly depend on the range of assumed values of $f_{*}$. From the discussion above we see that it should be at least from $f_{*} \simeq 0.01$ to $f_{*} \simeq 0.3$ (see, e.g., [78]). We see that for $f_{*}=0.09$, in both 7-keV sterile neutrinos and thermal relics with $m_{\mathrm{TR}}=6 \mathrm{keV}$, the minimum of $\delta T_{b}(z)$ happens around $z=17$, in agreement with the EDGES results. On the contrary, taking $f_{*}=0.03$ (as done in [43]) would make CDM consistent with the EDGES data, while the two WDM models would have an insufficient number of Lyman- $\alpha$ photons at the redshifts of interest.

\footnotetext{
${ }^{3}$ Lepton asymmetry $L_{6} \equiv 10^{6}\left(n_{\nu_{e}}-n_{\bar{\nu}_{e}}\right) / s$, where $n_{\nu_{e}}$ and $n_{\bar{\nu}_{e}}$ are the number densities of electron neutrinos and antineutrinos, and $s$ is the total entropy density in the early Universe [83,84].
} 


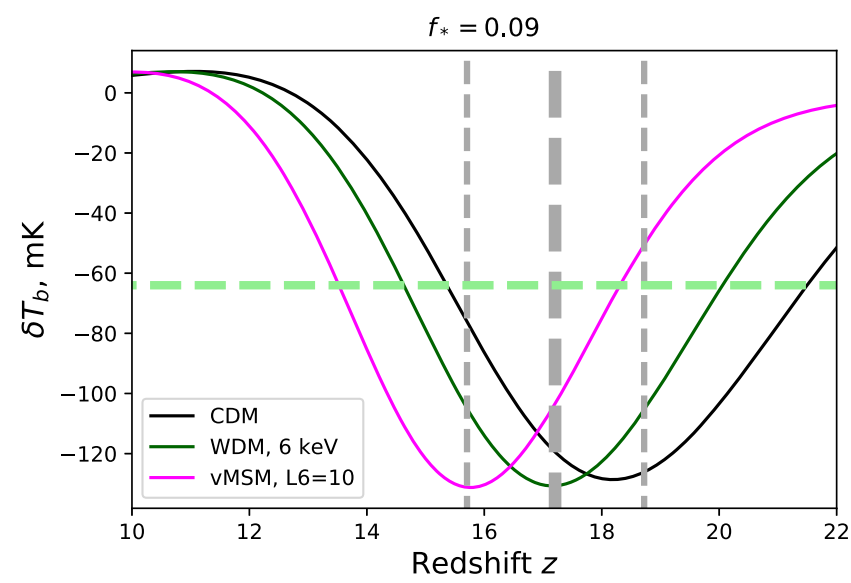

FIG. 2. $\delta T_{b}$ as a function of redshift $z$ for three models of interest: CDM, thermal-relic WDM with mass $m_{\mathrm{TH}}=6 \mathrm{keV}$, and resonantly produced sterile-neutrino DM with mass $7 \mathrm{keV}$ and lepton asymmetry $L_{6}=10$. For all models the minimal virial temperature of halos is fixed at $T_{\text {vir }}=10^{4} \mathrm{~K}$, corresponding to atomic hydrogen cooling; see, e.g., Fig. 12 and Eq. (26) of [45]. The stellar formation efficiency $f_{*}$ is chosen to be 0.09 . Due to higher star-formation efficiency as compared to, e.g., $[43,66]$, the position of the $21-\mathrm{cm}$ absorption trough becomes consistent with EDGES observations (indicated by the grey vertical lines) for all three models of our interest. The green horizontal line denotes half of the absorption depth; it is plotted in order to illustrate the full width at half maximum of the absorption troughs in the models of our interest.

In Fig. 3 we plot the range of $f_{*}$ 's that have the minimum of the absorption trough for $15.8 \leq z \leq 18.7$. We see that starting from $m_{\mathrm{TR}} \leq 4 \mathrm{keV}, f_{*}$ can be as large as $100 \%$ that for masses of this order or above. Given several orders of magnitude uncertainties in $f_{*}$ (as discussed above), the only robust bound can be obtained if one chooses $f_{*}=1$; at most all baryons enter star formation.

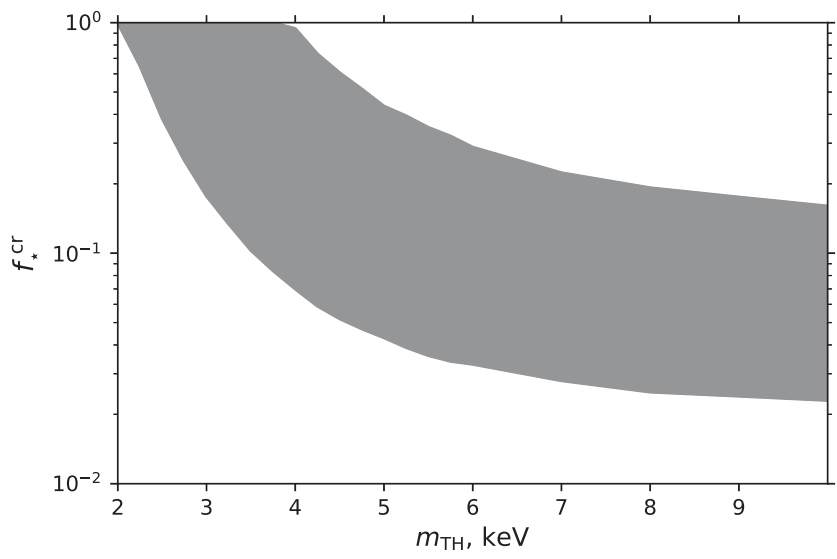

FIG. 3. The range of values of $f_{*}$ for which the minimum of the absorption trough lies in the redshift range $15.8 \leq z \leq 18.7$, consistent with EDGES observations. For all models the minimal virial temperature of halos is fixed at $T_{\text {vir }}=10^{4} \mathrm{~K}$, corresponding to atomic hydrogen cooling
In this case, for example, thermal WDM masses as light as $m_{\mathrm{TR}} \geq 2 \mathrm{keV}$ cannot be excluded (see Fig. 4). This puts the sensitivity of the EDGES signal in line with a number of previous bounds on WDM parameters (see, e.g., the Lyman- $\alpha$ constraints [93], taking into account proper marginalizations over possible thermal histories; bounds [98] from counting of high- $z$ galaxies; bounds $[99,100]$ from strong gravitational lensing; bounds [101,102] from the Milky Way satellite counts, etc.). As [103] demonstrates, future measurements of star-formation efficiency at high redshifts, as well as the $21-\mathrm{cm}$ power spectrum, are required to improve the sensitivity for WDM particles.

In this paper we have concentrated on the redshift position of the minimum of $\delta T_{b}(z)$ as an indicator of star-forming processes at high redshifts. However, both the depth of the 21-cm absorption trough and its width carry important information about the underlying physics.

Much like the position, the width of the obtained profile also depends on the cosmology. When using $T_{\text {vir }}=10^{3} \mathrm{~K}$ (molecular cooling) and ignoring possible suppression due to the Lyman-Werner radiation background (see, e.g., [104]), we see that CDM predicts an absorption-trough width which is larger than the one observed by the EDGES experiment, Fig. 5. For the WDM and $\nu$ MSM profiles, the molecular cooling brings little to no effect due to the lack of substructures of the mass $\sim M_{\min }$.

The depth of the observed trough is much greater than what any of the models discussed in this paper predict. To date, only additional, nongravitational, baryon-DM interactions can accommodate such a strong spin-temperature cooling, which is beyond the scope of this paper [22-24,106].

To summarize, we discussed the large uncertainty in star formation at very high redshifts $(z \sim 17)$, which are probed by recent EDGES observations of the global $21-\mathrm{cm}$ signal. As a consequence, using only this signal it is impossible to robustly constrain the parameters of dark matter models, such as the mass of the warm dark matter particle.

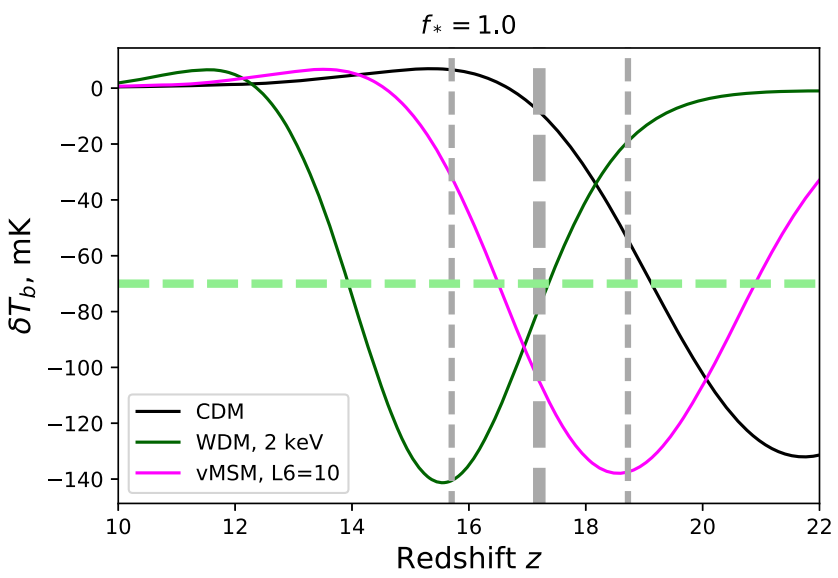

FIG. 4. The same as in Fig. 2 but for $f_{*}=1.0$. As we see, even the thermal WDM model with particle mass $m_{\mathrm{TH}}=2 \mathrm{keV}$ is consistent with observations. 

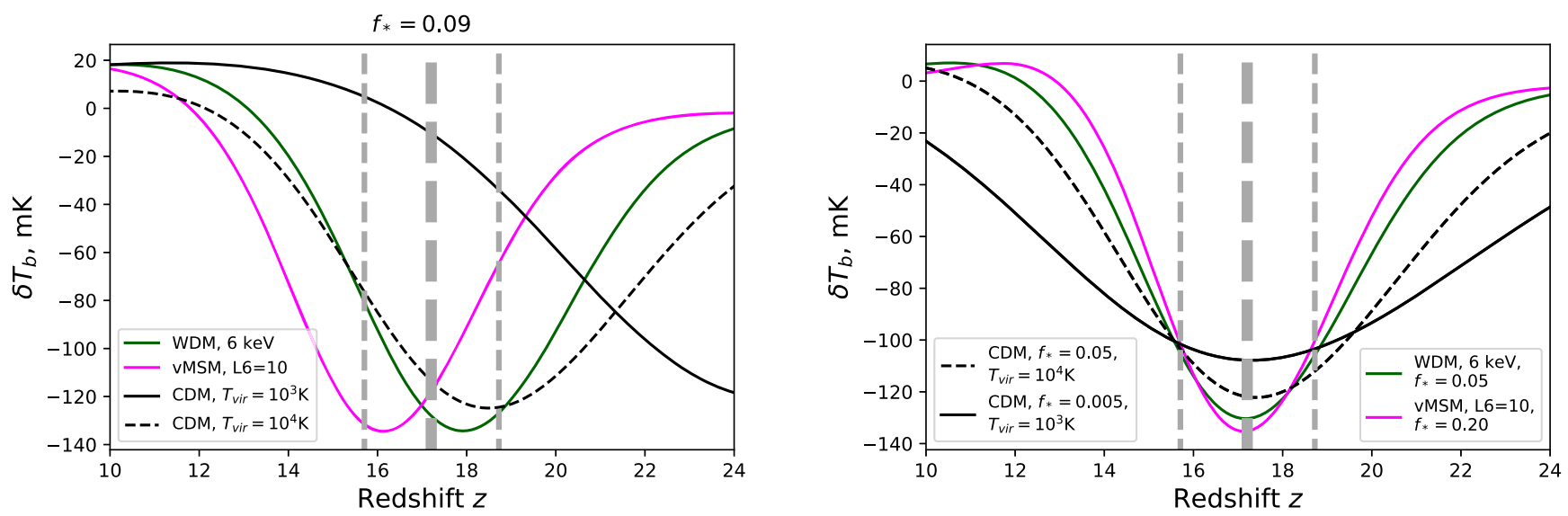

FIG. 5. Left panel: The same as in Fig. 2 but with minimal halo temperature $T_{\text {vir }}=10^{3} \mathrm{~K}$, which corresponds to molecular hydrogen cooling; see, e.g., [105] and Fig. 12 of [45] for details. By comparing with Fig. 2 we see that the decrease of $T_{\text {vir }}$ from $10^{4} \mathrm{~K}$ to $10^{3} \mathrm{~K}$ essentially does not change absorption profiles for 6-keV WDM and L6 $=10 \nu \mathrm{MSM}$ models. In contrast, in the CDM model, predictions change dramatically, both in profile width and position of the minimum (here, the black solid curve denotes $T_{\text {vir }}=10^{3} \mathrm{~K}$, while black dashed curve denotes $T_{\mathrm{vir}}=10^{4} \mathrm{~K}$ ), owing to a significant number of small mass halos (cf. [48]). Right panel: The same profiles as in the left panel but with values of $f_{*}$ adjusted to match the best-fit position of the EDGES absorption trough.

Conversely, various DM models need distinct star-formation scenarios to fit the signal. Detailed future studies of star formation at very high redshifts $(z \gtrsim 10)$, together with detailed modeling of structure assembly and early star formation, will reduce the existing uncertainties. Ongoing and future studies of the $21-\mathrm{cm}$ signal remain promising tools for inferring the key dark matter parameters.

\section{ACKNOWLEDGEMENTS}

We thank Tom Theuns for valuable comments on an earlier version of this paper and the authors of [48] for sharing with us results of their work before publication. The work of D.I. and O.R. was supported by the Carlsberg Foundation. The work of A. R. was partially supported by a grant for Young Scientists Research Laboratories of the National Academy of Sciences of Ukraine. A. R. also acknowledges the grant from the Abdus Salam International Centre for Theoretical Physics, Trieste, Italy. This project has received funding from the European Research Council (ERC) under the European Union's Horizon 2020 Research and Innovation Programme (GA 694896).
[1] A. H. Patil et al., Upper limits on the 21-cm epoch of reionization power spectrum from one night with LOFAR, Astrophys. J. 838, 65 (2017).

[2] B. K. Gehlot, F. G. Mertens, L. V. E. Koopmans, M. A. Brentjens, S. Zaroubi, B. Ciardi et al., The first power spectrum limit on the $21-\mathrm{cm}$ signal of neutral hydrogen during the cosmic dawn at $z=20-25$ from LOFAR, Mon. Not. R. Astron. Soc. 488, 4271 (2019).

[3] G. Paciga et al., A refined foreground-corrected limit on the HI power spectrum at $z=8.6$ from the GMRT epoch of reionization experiment, Mon. Not. R. Astron. Soc. 433, 639 (2013).

[4] Z. S. Ali et al., PAPER-64 constraints on reionization: The $21 \mathrm{~cm}$ power spectrum at $z=8.4$, Astrophys. J. 809, 61 (2015).

[5] Z. S. Ali, A. R. Parsons, H. Zheng, J. C. Pober, A. Liu, J.E. Aguirre et al., Erratum: PAPER64 constraints on reionization: The $21 \mathrm{~cm}$ power spectrum at $z=8.4$, Astrophys. J. 863, 201 (2018).
[6] A. Ewall-Wice, J. S. Dillon, A. Liu, and J. Hewitt, The impact of modelling errors on interferometer calibration for $21 \mathrm{~cm}$ power spectra, Mon. Not. R. Astron. Soc. 470, 1849 (2017).

[7] J. D. Bowman, A. E. E. Rogers, R. A. Monsalve, T. J. Mozdzen, and N. Mahesh, An absorption profile centred at 78 megahertz in the sky-averaged spectrum, Nature (London) 555, 67 (2018).

[8] R. Hills, G. Kulkarni, P. D. Meerburg, and E. Puchwein, Concerns about modelling of foregrounds and the $21-\mathrm{cm}$ signal in EDGES data, Nature (London) 564, E32 (2018).

[9] R. F. Bradley, K. Tauscher, D. Rapetti, and J. O. Burns, A ground plane artifact that induces an absorption profile in sveraged spectra from global $21-\mathrm{cm}$ measurements-with possible application to EDGES, Astrophys. J. 874, 153 (2019).

[10] D. R. DeBoer et al., Hydrogen epoch of reionization array (HERA), Publ. Astron. Soc. Pac. 129, 045001 (2017). 
[11] L. V. E. Koopmans et al., The cosmic dawn and epoch of reionization with the square kilometre array, Proc. Sci., AASKA14 (2015) 001 [arXiv:1505.07568].

[12] P. Bull et al., Fundamental physics with the square kilometer array, arXiv:1810.02680.

[13] M. McQuinn, O. Zahn, M. Zaldarriaga, L. Hernquist, and S. R. Furlanetto, Cosmological parameter estimation using $21 \mathrm{~cm}$ radiation from the epoch of reionization, Astrophys. J. 653, 815 (2006).

[14] Y. Mao, M. Tegmark, M. McQuinn, M. Zaldarriaga, and O. Zahn, How accurately can $21 \mathrm{~cm}$ tomography constrain cosmology?, Phys. Rev. D 78, 023529 (2008).

[15] Y. Oyama, K. Kohri, and M. Hazumi, Constraints on the neutrino parameters by future cosmological $21 \mathrm{~cm}$ line and precise CMB polarization observations, J. Cosmol. Astropart. Phys. 02 (2016) 008.

[16] G. D’Amico, P. Panci, and A. Strumia, Bounds on Dark Matter Annihilations from $21 \mathrm{~cm}$ Data, Phys. Rev. Lett. 121, 011103 (2018).

[17] Y. Yang, The contributions of dark matter annihilation to the global $21 \mathrm{~cm}$ spectrum observed by the EDGES experiment, Phys. Rev. D 98, 103503 (2018).

[18] S. Clark, B. Dutta, Y. Gao, Y.-Z. Ma, and L. E. Strigari, $21 \mathrm{~cm}$ limits on decaying dark matter and primordial black holes, Phys. Rev. D 98, 043006 (2018).

[19] H. Liu and T. R. Slatyer, Implications of a 21-cm signal for dark matter annihilation and decay, Phys. Rev. D 98, 023501 (2018).

[20] K. Cheung, J.-L. Kuo, K.-W. Ng, and Y.-L. S. Tsai, The impact of EDGES 21-cm data on dark matter interactions, Phys. Lett. B 789, 137 (2019).

[21] A. Mitridate and A. Podo, Bounds on dark matter decay from $21 \mathrm{~cm}$ line, J. Cosmol. Astropart. Phys. 05 (2018) 069.

[22] R. Barkana, Possible interaction between baryons and dark-matter particles revealed by the first stars, Nature (London) 555, 71 (2018).

[23] A. Fialkov, R. Barkana, and A. Cohen, Constraining Baryon-Dark Matter Scattering with the Cosmic Dawn 21-cm Signal, Phys. Rev. Lett. 121, 011101 (2018).

[24] A. Berlin, D. Hooper, G. Krnjaic, and S. D. McDermott, Severely Constraining Dark Matter Interpretations of the 21-cm Anomaly, Phys. Rev. Lett. 121, 011102 (2018).

[25] R. Barkana, N. J. Outmezguine, D. Redigolo, and T. Volansky, Strong constraints on light dark matter interpretation of the EDGES signal, Phys. Rev. D 98, 103005 (2018).

[26] S. Fraser et al., The EDGES $21 \mathrm{~cm}$ anomaly and properties of dark matter, Phys. Lett. B 785, 159 (2018).

[27] T. R. Slatyer and C.-L. Wu, Early-Universe constraints on dark matter-baryon scattering and their implications for a global 21 cm signal, Phys. Rev. D 98, 023013 (2018).

[28] P. Madau, A. Meiksin, and M. J. Rees, 21-cm tomography of the intergalactic medium at high redshift, Astrophys. J. 475, 429 (1997).

[29] S. Furlanetto, S. P. Oh, and F. Briggs, Cosmology at low frequencies: The $21 \mathrm{~cm}$ transition and the high-redshift Universe, Phys. Rep. 433, 181 (2006).

[30] M. Zaldarriaga, S. R. Furlanetto, and L. Hernquist, 21 Centimeter fluctuations from cosmic gas at high redshifts, Astrophys. J. 608, 622 (2004).
[31] B. Ciardi and A. Ferrara, The first cosmic structures and their effects, Space Sci. Rev. 116, 625 (2005).

[32] J. R. Pritchard and A. Loeb, 21-cm cosmology, Rep. Prog. Phys. 75, 086901 (2012).

[33] R. Barkana, The rise of the first stars: Supersonic streaming, radiative feedback and 21-cm cosmology, Phys. Rep. 645, 1 (2016).

[34] J. R. Pritchard and A. Loeb, Evolution of the $21 \mathrm{~cm}$ signal throughout cosmic history, Phys. Rev. D 78, 103511 (2008).

[35] J. Mirocha, Decoding the x-ray properties of prereionization era sources, Mon. Not. R. Astron. Soc. 443, 1211 (2014).

[36] A. Mesinger, S. Furlanetto, and R. Cen, 21 cmFAST: A fast, semi-numerical simulation of the high-redshift $21-\mathrm{cm}$ signal, Mon. Not. R. Astron. Soc. 411, 955 (2011).

[37] J. R. Bond, G. Efstathiou, and J. Silk, Massive Neutrinos and the Large Scale Structure of the Universe, Phys. Rev. Lett. 45, 1980 (1980).

[38] A. Boyarsky, J. Lesgourgues, O. Ruchayskiy, and M. Viel, Lyman- $\alpha$ constraints on warm and on warm-plus-cold dark matter models, J. Cosmol. Astropart. Phys. 05 (2009) 012.

[39] A. Boyarsky, M. Drewes, T. Lasserre, S. Mertens, and O. Ruchayskiy, Sterile neutrino dark matter, Prog. Part. Nucl. Phys. 104, 1 (2019).

[40] S. Dodelson and L. M. Widrow, Sterile-Neutrinos as Dark Matter, Phys. Rev. Lett. 72, 17 (1994).

[41] M. Viel, J. Lesgourgues, M. G. Haehnelt, S. Matarrese, and A. Riotto, Constraining warm dark matter candidates including sterile neutrinos and light gravitinos with WMAP and the Lyman- $\alpha$ forest, Phys. Rev. D 71, 063534 (2005).

[42] A. J. Benson, A. Farahi, S. Cole, L. A. Moustakas, A. Jenkins, M. Lovell, R. Kennedy, J. Helly, and C. Frenk, Dark matter halo merger histories beyond cold dark matter: I-Methods and application to warm dark matter, Mon. Not. R. Astron. Soc. 428, 1774 (2013).

[43] A. Schneider, Constraining non-cold dark matter models with the global 21-cm signal, Phys. Rev. D 98, 063021 (2018).

[44] Z. Haiman, T. Abel, and M. J. Rees, The radiative feedback of the first cosmological objects, Astrophys. J. 534, 11 (2000).

[45] R. Barkana and A. Loeb, In the beginning: The first sources of light and the reionization of the Universe, Phys. Rep. 349, 125 (2001).

[46] L. Gao and T. Theuns, Lighting the Universe with filaments, Science 317, 1527 (2007).

[47] S. Paduroiu, Y. Revaz, and D. Pfenniger, Structure formation in warm dark matter cosmologies: Top-bottom upside-down, arXiv:1506.03789.

[48] M. Leo, T. Theuns, C. M. Baugh, B. Li, and S. Pascoli, Constraining structure formation using EDGES, arXiv: 1909.04641.

[49] A. Chatterjee, P. Dayal, T. R. Choudhury, and A. Hutter, Ruling out $3 \mathrm{keV}$ warm dark matter using $21 \mathrm{~cm}$-EDGES data, Mon. Not. R. Astron. Soc. 487, 3560 (2019).

[50] L. Lopez-Honorez, O. Mena, and P. Villanueva-Domingo, Dark matter microphysics and $21 \mathrm{~cm}$ observations, Phys. Rev. D 99, 023522 (2019).

[51] L. Gao, T. Theuns, and V. Springel, Star forming filaments in warm dark matter models, Mon. Not. R. Astron. Soc. 450, 45 (2015). 
[52] J. Herpich, G. S. Stinson, A. V. Macci, C. Brook, J. Wadsley, H. M. P. Couchman, and T. Quinn, MaGICCWDM: The effects of warm dark matter in hydrodynamical simulations of disc galaxy formation, Mon. Not. R. Astron. Soc. 437, 293 (2014).

[53] U. Maio and M. Viel, The first billion years of a warm dark matter Universe, Mon. Not. R. Astron. Soc. 446, 2760 (2015).

[54] P. Colin, V. Avila-Reese, A. Gonzalez-Samaniego, and H. Velazquez, Simulations of galaxies formed in warm dark matter halos of masses at the filtering scale, Astrophys. J. 803, 28 (2015).

[55] C. Power and A. S. G. Robotham, The extended stellar component of Galaxies the nature of dark matter, Astrophys. J. 825, 31 (2016).

[56] M. R. Lovell et al., Properties of local group galaxies in hydrodynamical simulations of sterile neutrino dark matter cosmologies, Mon. Not. R. Astron. Soc. 468, 4285 (2017).

[57] N. Menci, F. Fiore, and A. Lamastra, Galaxy formation in WDM cosmology, Mon. Not. R. Astron. Soc. 421, 2384 (2012).

[58] X. Kang, A. V. Maccio, and A. A. Dutton, The effect of warm dark matter on galaxy properties: Constraints from the stellar mass function and the Tully-Fisher relation, Astrophys. J. 767, 22 (2013).

[59] N. Menci, F. Fiore, and A. Lamastra, The evolution of active Galactic nuclei in warm dark matter cosmology, Astrophys. J. 766, 110 (2013).

[60] A. M. Nierenberg, T. Treu, N. Menci, Y. Lu, and W. Wang, The cosmic evolution of faint satellite Galaxies as a test of Galaxy formation and the nature of dark matter, Astrophys. J. 772, 146 (2013).

[61] M. R. Lovell, S. Bose, A. Boyarsky, S. Cole, C. S. Frenk, V. Gonzalez-Perez, R. Kennedy, O. Ruchayskiy, and A. Smith, Satellite galaxies in semi-analytic models of galaxy formation with sterile neutrino dark matter, Mon. Not. R. Astron. Soc. 461, 60 (2016).

[62] M. R. Lovell, V. Gonzalez-Perez, S. Bose, A. Boyarsky, S. Cole, C. S. Frenk, and O. Ruchayskiy, Addressing the too big to fail problem with baryon physics and sterile neutrino dark matter, Mon. Not. R. Astron. Soc. 468, 2836 (2017).

[63] L. Wang, V. Gonzalez-Perez, L. Xie, A. P. Cooper, C. S. Frenk, L. Gao, W. A. Hellwing, J. Helly, M. R. Lovell, and L. Jiang, The galaxy population in cold and warm dark matter cosmologies, Mon. Not. R. Astron. Soc. 468, 4579 (2017).

[64] S. Bose, C. S. Frenk, J. Hou, C. G. Lacey, and M. R. Lovell, Reionization in sterile neutrino cosmologies, Mon. Not. R. Astron. Soc. 463, 3848 (2016).

[65] H. E. Ross, K. L. Dixon, R. Ghara, I. T. Iliev, and G. Mellema, Evaluating the QSO contribution to the 21-cm signal from the cosmic dawn, Mon. Not. R. Astron. Soc. 487, 1101 (2019).

[66] M. Safarzadeh, E. Scannapieco, and A. Babul, A limit on the warm dark matter particle mass from the redshifted $21 \mathrm{~cm}$ absorption line, Astrophys. J. 859, L18 (2018).

[67] G. L. Bryan and M. L. Norman, Statistical properties of X-ray clusters: Analytic and numerical comparisons, Astrophys. J. 495, 80 (1998).
[68] P. A. Oesch, R. J. Bouwens, G. D. Illingworth, I. Labbé, and M. Stefanon, The dearth of $z \sim 10$ Galaxies in all HST legacy fields - The rapid evolution of the Galaxy population in the first 500 Myr, Astrophys. J. 855, 105 (2018).

[69] P. Dayal, A. Ferrara, J. S. Dunlop, and F. Pacucci, Essential physics of early galaxy formation, Mon. Not. R. Astron. Soc. 445, 2545 (2014).

[70] G. Sun and S. R. Furlanetto, Constraints on the star formation efficiency of galaxies during the epoch of reionization, Mon. Not. R. Astron. Soc. 460, 417 (2016).

[71] J. Mirocha, S. R. Furlanetto, and G. Sun, The global 21-cm signal in the context of the high- $z$ galaxy luminosity function, Mon. Not. R. Astron. Soc. 464, 1365 (2017).

[72] J. Mirocha and S. R. Furlanetto, What does the first highlyredshifted 21-cm detection tell us about early galaxies?, Mon. Not. R. Astron. Soc. 483, 1980 (2019).

[73] J. Park, A. Mesinger, B. Greig, and N. Gillet, Inferring the astrophysics of reionization and cosmic dawn from galaxy luminosity functions and the 21-cm signal, Mon. Not. R. Astron. Soc. 484, 933 (2019).

[74] T. Sawala et al., Bent by baryons: The low mass galaxyhalo relation, Mon. Not. R. Astron. Soc. 448, 2941 (2015).

[75] P. S. Corasaniti, S. Agarwal, D. J. E. Marsh, and S. Das, Constraints on dark matter scenarios from measurements of the galaxy luminosity function at high redshifts, Phys. Rev. D 95, 083512 (2017).

[76] N. Menci, A. Grazian, A. Lamastra, F. Calura, M. Castellano, and P. Santini, Galaxy formation in sterile neutrino dark matter models, Astrophys. J. 854, 1 (2018).

[77] J. H. Wise, V. G. Demchenko, M. T. Halicek, M. L. Norman, M. J. Turk, T. Abel, and B. D. Smith, The birth of a galaxy III. Propelling reionization with the faintest galaxies, Mon. Not. R. Astron. Soc. 442, 2560 (2014).

[78] H. Xu, J. H. Wise, M. L. Norman, K. Ahn, and B. W. O'Shea, Galaxy properties and UV escape fractions during the epoch of reionization: Results from the renaissance simulations, Astrophys. J. 833, 84 (2016).

[79] X. Ma, P. F. Hopkins, S. Garrison-Kimmel, C.-A. FaucherGigure, E. Quataert, M. Boylan-Kolchin, C. C. Hayward, R. Feldmann, and D. Kereš, Simulating galaxies in the reionization era with FIRE-2: Galaxy scaling relations, stellar mass functions, and luminosity functions, Mon. Not. R. Astron. Soc. 478, 1694 (2018).

[80] J. Rosdahl, H. Katz, J. Blaizot, T. Kimm, L. MichelDansac, T. Garel, M. Haehnelt, P. Ocvirk, and R. Teyssier, The SPHINX cosmological simulations of the first billion years: The impact of binary stars on reionization, Mon. Not. R. Astron. Soc. 479, 994 (2018).

[81] M. Sharma and T. Theuns, The I $\epsilon \alpha$ model of feedbackregulated galaxy formation, arXiv:1906.10135.

[82] P. Dayal and A. Ferrara, Early galaxy formation and its large-scale effects, Phys. Rep. 780-782, 1 (2018).

[83] M. Laine and M. Shaposhnikov, Sterile neutrino dark matter as a consequence of nuMSM-induced lepton asymmetry, J. Cosmol. Astropart. Phys. 06 (2008) 031.

[84] A. Boyarsky, O. Ruchayskiy, and M. Shaposhnikov, The role of sterile neutrinos in cosmology and astrophysics, Annu. Rev. Nucl. Part. Sci. 59, 191 (2009).

[85] E. Bulbul, M. Markevitch, A. Foster, R. K. Smith, M. Loewenstein, and S.W. Randall, Detection of an 
unidentified emission line in the stacked x-ray spectrum of Galaxy clusters, Astrophys. J. 789, 13 (2014).

[86] A. Boyarsky, O. Ruchayskiy, D. Iakubovskyi, and J. Franse, Unidentified Line in X-Ray Spectra of the Andromeda Galaxy and Perseus Galaxy Cluster, Phys. Rev. Lett. 113, 251301 (2014).

[87] A. Boyarsky, J. Franse, D. Iakubovskyi, and O. Ruchayskiy, Checking the Dark Matter Origin of a $3.53 \mathrm{keV}$ Line with the Milky Way Center, Phys. Rev. Lett. 115, 161301 (2015).

[88] D. Iakubovskyi, E. Bulbul, A. R. Foster, D. Savchenko, and V. Sadova, Testing the origin of $3.55 \mathrm{keV}$ line in individual galaxy clusters observed with XMM-Newton, arXiv:1508.05186.

[89] O. Ruchayskiy, A. Boyarsky, D. Iakubovskyi, E. Bulbul, D. Eckert, J. Franse, D. Malyshev, M. Markevitch, and A. Neronov, Searching for decaying dark matter in deep XMMNewton observation of the Draco dwarf spheroidal, Mon. Not. R. Astron. Soc. 460, 1390 (2016).

[90] J. Franse et al., Radial profile of the $3.55 \mathrm{keV}$ line out to $R_{200}$ in the Perseus cluster, Astrophys. J. 829, 124 (2016).

[91] M. Drewes et al., A white paper on $\mathrm{keV}$ sterile neutrino dark matter, J. Cosmol. Astropart. Phys. 01 (2017) 025.

[92] K. N. Abazajian, Sterile neutrinos in cosmology, Phys. Rep. 711-712, 1 (2017).

[93] A. Garzilli, A. Boyarsky, and O. Ruchayskiy, Cutoff in the Lyman-alpha forest power spectrum: Warm IGM or warm dark matter?, Phys. Lett. B 773, 258 (2017).

[94] J. Baur, N. Palanque-Delabrouille, C. Yeche, A. Boyarsky, O. Ruchayskiy, É. Armengaud, and J. Lesgourgues, Constraints from Ly- $\alpha$ forests on non-thermal dark matter including resonantly-produced sterile neutrinos, J. Cosmol. Astropart. Phys. 12 (2017) 013.

[95] A. Garzilli, A. Magalich, T. Theuns, C. S. Frenk, C. Weniger, O. Ruchayskiy, and A. Boyarsky, The Lyman$\alpha$ forest as a diagnostic of the nature of the dark matter, Mon. Not. R. Astron. Soc. 489, 3456 (2019).
[96] A. Rudakovskiy and D. Iakubovskyi, Influence of $7 \mathrm{keV}$ sterile neutrino dark matter on the process of reionization, J. Cosmol. Astropart. Phys. 06 (2016) 017.

[97] A. Rudakovskyi and D. Iakubovskyi, Dark matter model favoured by reionization data: $7 \mathrm{keV}$ sterile neutrino vs cold dark matter, Mon. Not. R. Astron. Soc. 483, 4080 (2019).

[98] N. Menci, A. Grazian, M. Castellano, and N. G. Sanchez, A stringent limit on the warm dark matter particle masses from the abundance of $z=6$ Galaxies in the hubble frontier fields, Astrophys. J. 825, L1 (2016).

[99] S. Birrer, A. Amara, and A. Refregier, Lensing substructure quantification in RXJ1131-1231: A $2 \mathrm{keV}$ lower bound on dark matter thermal relic mass, J. Cosmol. Astropart. Phys. 05 (2017) 037.

[100] S. Vegetti, G. Despali, M. R. Lovell, and W. Enzi, Constraining sterile neutrino cosmologies with strong gravitational lensing observations at redshift $z \sim 0.2$, Mon. Not. R. Astron. Soc. 481, 3661 (2018).

[101] M. R. Lovell, C. S. Frenk, V. R. Eke, A. Jenkins, L. Gao, and $\mathrm{T}$. Theuns, The properties of warm dark matter haloes, Mon. Not. R. Astron. Soc. 439, 300 (2014).

[102] R. Kennedy, C. Frenk, S. Cole, and A. Benson, Constraining the warm dark matter particle mass with Milky Way satellites, Mon. Not. R. Astron. Soc. 442, 2487 (2014).

[103] A. Mesinger, A. Ferrara, and D. S. Spiegel, Signatures of $\mathrm{X}$-rays in the early Universe, Mon. Not. R. Astron. Soc. 431, 621 (2013).

[104] B. Yue and X. Chen, Reionization in the warm dark matter model, Astrophys. J. 747, 127 (2012).

[105] D. Galli and F. Palla, The chemistry of the early universe, Astron. Astrophys. 335, 403 (1998).

[106] J. B. Muoz and A. Loeb, A small amount of mini-charged dark matter could cool the baryons in the early Universe, Nature (London) 557, 684 (2018). 